\title{
Revitalizing an Electromechanical Energy Conversion Course
}

\section{Thomas E McDermott P.E., University of Pittsburgh}

Thomas E. McDermott is an Assistant Professor at the University of Pittsburgh, with over 30 years of industrial experience in consulting and software development. His research interests include electric power distribution systems, renewable energy, power electronics, electromagnetics, and circuit simulation. Tom is a registered professional engineer in Pennsylvania and an IEEE Fellow. He has a B. S. and M. Eng. in Electric Power from Rensselaer, and a Ph.D. in Electrical Engineering from Virginia Tech.

\section{Dr. Renee M Clark, University of Pittsburgh}

Renee Clark serves as the Director of Assessment for the Swanson School of Engineering at the University of Pittsburgh. She received her PhD from the Department of Industrial Engineering, where she also completed her post-doctoral studies. Her research has primarily focused on the application of data analysis techniques to engineering education research studies as well as industrial accidents. She has over 20 years of experience in various engineering, IT, and data analysis positions within academia and industry, including ten years of manufacturing experience at Delphi Automotive. 


\section{Revitalizing an Electromechanical Energy Conversion Course}

Our University's Electrical and Computer Engineering Department has offered an elective course in "Electric Machinery" for decades. It is a 4-credit course offered each fall term for juniors and seniors, with a laboratory component. Prior to fall 2013, this course had been lectureheavy due to school scheduling requirements, and it suffered from use of old laboratory equipment that was difficult to maintain. With increasing focus on renewable energy and power electronics in the curriculum, we felt the need to modernize this course so that it provides a better learning experience and appeals to more students.

Over a period of two terms, we have implemented several improvements to the lab work. The original seven hardware lab experiments were replaced with six new hardware experiments and six new computer software labs. Thus, the course's hands-on laboratory content nearly doubled. The new hardware experiments feature power electronics, new machines and new instrumentation. The new computer modeling assignments feature finite element and multidomain system simulation software.

In the fall 2014 term, we have also "flipped" the course. Each week, students watch approximately 40 minutes of video, broken into segments. Most of the videos are screencasts that introduce new concepts or illustrate example problems in advance of the lecture; those are typically four to eight minutes in length. A few of the videos are longer; they were made with Adobe Captivate to demonstrate software operation in advance of the computer labs. This frees the lecture time for more interactive work, including problem sets worked in teams, questionand-answer periods, and either a hardware or computer lab session. The video segments also allow students to review concepts on their own schedules, as they work through assigned textbook readings and problems. The course grading criteria consists of $80 \%$ in-class activity, namely the problem sets and lab assignments, plus $20 \%$ final exam. There have been essentially no unexcused absences from class.

The classroom environment was evaluated through the University Student Opinion of Teaching Survey. In fall 2013, the results encouraged further development of both hardware and software labs, which we continued in fall 2014. Course enrollment also increased by $60 \%$ in fall 2014 , from 13 to 21 students. Favorable teaching evaluations and comments from fall 2013 encouraged even more classroom interaction, which led us to the "flipping" model. The fall 2014 course has been evaluated with a new Teaching Survey, supplemented with custom questions about the flipped classroom. We further evaluated this flipped classroom for the degree of instructorsupported active learning and problem solving and student interaction, and for impact on student final exam performance. The results were mixed, and we discuss plans for future course offerings that will retain favorable elements of the flipped classroom. 


\section{The Place of this Course in our Electric Power Concentration}

Our department offers four concentration areas to EE majors, and approximately one third of them choose the Electric Power concentration ${ }^{1}$. (The other concentration areas include Digital Systems, Electronics and Devices, and Communications and Signal Processing. Computer Engineering is a separate major offered within our department.) In order to complete the Electric Power concentration, students must take Power System Analysis 1 and three electives, chosen from:

1. Linear Control Systems (a required choice now, becomes optional in fall 2015)

2. Electric Machinery (i.e. this course)

3. Power Generation, Operation and Control

4. Power Distribution Systems Engineering and Smart Grids

5. Construction and Cost of Electrical Supply

6. Introduction to Thermodynamics

7. Introduction to Nuclear Engineering

8. Power Electronics (a graduate course open to seniors)

The ABET outcomes to be addressed in this course include:

a. Ability to apply knowledge of mathematics, science and engineering

b. Ability to design and construct experiments, as well as to analyze and interpret data

e. Ability to identify, formulate and solve engineering problems

f. Understanding of professional and ethical responsibility

1. Ability to use the techniques, skills and modern engineering tools necessary for engineering practice

Although not required, this course has been a popular elective within the concentration area because it's the only one offering a lab experience in electric power. As such, at carries 4 credits while the others all carry 3 credits.

The existing power electronics course carries a Master's-level course number, and few of our seniors take it. In department exit surveys of graduating seniors, this lack of a junior-senior level power electronics course has been pointed out consistently.

\section{Syllabus Changes in 2013 and 2014}

This course has been offered every fall term for many years. Prior to 2013, the course covered a sequence of traditional topics: magnetic circuits, three-phase transformers, DC machines, induction machines, and synchronous machines ${ }^{2}$. The class met for 140 minutes one evening per week. Seven lab assignments, detailed later, were scheduled for completion at different times in the week. The machines $l a b^{3}$ had facilities for only one student team to work at a time. 
In 2013, the machines lab became unavailable due to building renovation, and at the same time, we were constructing a new electric power $\mathrm{lab}^{4}$. We also wanted to address the gap in power electronics education, at least until a more comprehensive solution could be implemented. This offered both motivation and opportunity to revamp the electric machinery course. In fall 2013, the syllabus was refined to cover the weekly topics shown in Table 1, and the textbook was changed to incorporate more power electronics content ${ }^{5}$.

Table 1: Course Topics in 2013 and 2014

\begin{tabular}{|c|l|l|}
\hline Week & Fall 2013 Topic (13 Students) & Fall 2014 Topic (21 Students) \\
\hline 1 & Three-phase power and harmonics & \multicolumn{2}{|l|}{} \\
\hline 2 & Magnetic circuits & Transformers and the per-unit system \\
\hline 3 & Transformers & Transformer connections \\
\hline 4 & $\begin{array}{l}\text { Principles of finite element and circuit } \\
\text { simulation software (guest lecture) }\end{array}$ & \multicolumn{2}{|l|}{} \\
\hline 5 & Mechanical load systems & \\
\hline 6 & Induction machines I - equivalent circuits and tests \\
\hline 7 & Induction machines II - performance \\
\hline 8 & Induction motor speed control and power electronics devices \\
\hline 9 & Three-phase AC motor drives & Specialty motors / software tools \\
\hline 10 & Polyphase synchronous machines I - equivalent circuits and tests \\
\hline 11 & Polyphase synchronous machines II - performance \\
\hline 12 & DC machines & DC machines \\
\hline 13 & Principles of electric power measurements \\
\hline 14 & DC motor drives
\end{tabular}

There were 6 computer lab assignments and 1 hardware lab assignments in fall 2013, and the hardware lab session was actually conducted before our new lab's formal opening in January 2014. In student course evaluations, the response was very positive to all of the new course elements. Therefore, only a few changes were made to the topic outline for fall 2014. In week 4, the guest lecture on software tools was shortened and postponed till week 12 . The extra time early in the course was used for better coverage of three-phase transformer connections. In week 13 of fall 2013, the students performed one lab experiment during class time. In fall 2014, the experiments did not require full class periods, so the extra time was used to cover universal motors and brushless DC machines, which had not been covered in 2013.

The 2013 students did complain about the long evening class periods, and the pace of material covered in only one meeting per week. The instructor thought the course could be improved with more interaction, more hands-on work in the lab, and by distributing student learning efforts throughout the week. For 2014, the number of lab assignments approximately doubled, and the 
length of in-class lecture approximately halved. The instructor had some positive experience with video lecture and software demo recordings in a spring 2014 graduate course, and had taken a University-sponsored seminar on flipped classrooms in fall 2013. It was felt that flipping the 2014 class could be helpful in breaking up the long evening sessions, and in providing material for students to review throughout the week. The course evaluations from 2013 were already high, and improving them was not a specific goal.

\section{Literature Review of Other Flipped EE Classrooms}

Upon a review of the literature, we found other electrical engineering courses that have been flipped nationally, with mostly positive results. In a signal processing course at the University of Wisconsin, some students "missed" the traditional lecture format initially. The instructor noted that it took a few weeks for the students to adapt to the new environment, engage with their peers, or ask for assistance. However, by the end of the term, fewer than $10 \%$ of the students indicated a preference for the conventional lecture format ${ }^{6}$. In addition, the signal processing instructor noted a very clear improvement in student achievement with the flipped classroom, with an overwhelming majority of students performing at a high level on the final exam, as never seen before. On this exam, a very small percentage of students scored below 70\%. The instructor likewise felt that the students came to class prepared for active learning. Based on his experiences, he concluded that flipped instruction made a very significant contribution to student learning gains and that his course was successful ${ }^{6}$.

However, an instructor at John Brown University in Arkansas noted a high level of frustration in his flipped sophomore electrical engineering course near the end of the term when students struggled to understand certain concepts. A group of students asked, "Are you ever going to lecture prior to assigning problems? We need the lecture because we are lost." He expressed caution about using the flipped method for all subjects. In particular, he indicated that for complex topics, it may be necessary to have mini-lectures to ensure a proper foundation ${ }^{7}$.

Similar to the experience at John Brown University, students' perspective towards the flipped classroom in the Principles of Electrical Engineering course at Texas A\&M International University in Laredo, Texas became less favorable near the end of the semester. At this point, the material became more challenging and difficult to understand independently. In the middle of the semester, $67 \%$ of survey respondents indicated they wanted to continue with the flipped format for the rest of the semester. However, by the end of the semester, just $57 \%$ would prefer the flipped format for this course if taken again ${ }^{8}$.

In a required junior-level electromagnetics course at the University of Utah, the instructor noted that students asked many more questions, including higher-thought-level questions, with the flipped format. Although exam scores showed no significant differences using the flipped format, the instructor felt that students achieved a higher level of learning, better understanding, and better problem solving skills. In addition, student evaluations of the course were higher with 
the flipped approach. Not only were the students supportive of this approach, but the instructor was highly pleased with the outcomes in her classroom ${ }^{9}$.

The flipped classroom has also been used in the Electronic Systems Engineering program at the University of Regina in Saskatchewan, Canada to enhance retention of lecture information and material. In a student survey in the Power Systems course, $62 \%$ of respondents found the flipped approach more useful than conventional lecture for presentation of material, and $80 \%$ felt that well-designed in-class assignments were a better use of class time ${ }^{10}$.

\section{Flipping from 2013 to 2014}

Table 2 shows the course grading criteria for each year evaluated. Letter grade criteria were the same each year; the instructor issued +/- grades both years. Each week included either a quiz or in-class problem set. In 2013, students were allowed to "drop" more in-class quiz grades, but they had to work alone on these quizzes and the quizzes were closed-book. In 2014, students worked in teams of two on timed but open-book problem sets. There was also a larger body of lab work to do in 2014. Homework was not collected in either year.

Table 2: Course Grading Criteria

\begin{tabular}{|l|l|}
\hline Fall 2013 & Fall 2014 \\
\hline 30\% Lab Reports (best 6 of 7) & 40\% Lab Reports (best 11 of 12) \\
\hline $\begin{array}{l}\text { 50\% In-class Closed-book Quizzes } \\
\text { (best 10 of 13) }\end{array}$ & $\begin{array}{l}\text { 40\% In-class Open-book Problem Sets } \\
\text { (best 12 of 13) }\end{array}$ \\
\hline 20\% Final Exam (formula sheet allowed) \\
\hline
\end{tabular}

In 2013, each class began with an open question-answer period. When the questions stopped, the quiz began. Following the quiz and a short break, the instructor presented new material with lecture and examples. The class usually ended after 2:30 or 2:45 hours, compared to the scheduled 3:30 hours, in recognition of the fact that students still had to complete 6 or 7 lab assignments outside of class time during the term. It was still a long evening for everyone. Textbook problems were suggested each week to help students prepare for the weekly quizzes, but these were not collected or monitored in any way.

In 2014, each class began with a short question-answer period, and then the problem set was passed out. Students could work together on these and consult their textbooks. Some of them were observed reviewing course video segments (described later) on their phones or tablets during the problem session - this was allowed. Following the problem session and a short break, the instructor presented new material with lecture and examples but for only 45 minutes, less than in 2013. Following this lecture, the whole class moved to either a department computer lab or the new electric power lab for that week's lab assignment. 
The 2014 sequence of topic introduction, review and evaluation was interleaved, as shown in Table 3. The instructor attempted to tailor this scheme for a one meeting per week format. There would have been less overlap in a two meeting per week format.

Table 3: Interleaving Textbook, Lecture and Video Segments in 2014 for Two Topics

\begin{tabular}{|c|l|l|}
\hline Week & Magnetic Circuits Topic & Transformers and Per-Unit System Topic \\
\hline 1 & Assigned Textbook Reading & Assigned Textbook Reading \\
\hline 2 & $\begin{array}{l}\text { In-class Lecture, assigned Video } \\
\text { Segments and practice problems }\end{array}$ & $\begin{array}{l}\text { In-class Lecture, assigned Video Segments and } \\
\text { practice problems }\end{array}$ \\
\hline 3 & In-class Problem Set & In-class Problem Set \\
\hline 4 & & \\
\hline
\end{tabular}

In both terms, the instructor mainly used a whiteboard for lectures and example problems. There was not a document camera available in the assigned classroom. In other courses, the instructor has found that a document camera enables the written class record to be scanned and uploaded to our learning management system after each class, and these notes are very helpful to students. In this case, the instructor did employ software demonstrations in many of the lectures ${ }^{11,12}$. PowerPoint slides were also used in a few limited instances.

\section{Video Segments in Fall 2014}

The course video content comprised 8:45:57 hours in 75 segments, all posted on a YouTube channel $^{13}$ and organized into weekly playlists. The average length of a video was 7:01, and the average length of a weekly playlist was 40:27. Most of the video segments were designed to present a specific topic, either based on the textbook or in some cases providing supplemental coverage. These "topic segments" were close to the average length of seven minutes, and they were done using a screen-cast approach using low-cost tools ${ }^{14,15,16}$. Some of the topic segments used software to demonstrate examples; these were also close to the average length but were made using University-licensed software demo capture software ${ }^{17}$. In response to student feedback, three of the latter playlists included 16 example problems, also close to the average length of seven minutes.

Two of the segments were designed to present demonstration walk-throughs of the software simulation programs used in the computer lab assignments ${ }^{18}$. Those two segments were longer, at 19:16 and 20:32. In fall 2013, both of these walk-throughs had been done in class.

After the first few weeks, these video segments became relatively easy to produce. It proved necessary to accept a less aggressive threshold for "re-takes"; technical errors need to be corrected, but most delivery errors need not be corrected. The overall preparation time probably exceeds the time to prepare an equivalent lecture presentation of the same material. There is an 
important advantage to the student in being able to pause and rewind the video at will. Because of this feature, the instructor does not have to cover every algebraic detail in example problems. Most if not all of the video segments could be reused in future course offerings, although the instructor would review each one before using it again (as with any previously developed course material.)

YouTube does keep track of the viewer count for each posted video. During the course term, it was apparent that only 50 to $75 \%$ of the class viewed every video segment before the upcoming problem session. The instructor expected that if students read the text and watched the videos, they would have the material needed to solve the problems. During the problem sessions, some of the students did review video segments that were directly pertinent to the assigned problems. Since the class ended, external subscribers to the YouTube channel have increased the viewer counts for most of the segments. These are copyrighted and licensed under a Creative Commons Attribution-Share Alike 4.0 International License.

\section{Hardware Lab Assignments}

Prior to 2013, the lab assignments included:

1. Transformers

2. Characteristics of a Synchronous Generator

3. Synchronous Motor

4. Characteristics of a Wound-Rotor Induction Motor

5. Characteristics of a DC Shunt Motor

6. Characteristics of a DC Series Motor

7. Singe-Phase Induction Motor

While providing valuable hands-on experience, the old lab equipment is inherently limited to connection, operation and measurement of machines. Machine design experiments are not really possible with the old lab equipment. The instrumentation consisted of analog voltmeters, ammeters and wattmeters. There were no modern power electronics experiments, while $28 \%$ of the content focused on DC machines that are less important today.

A new Electric Power Systems Lab (EPSL) at our University opened in January 2014 for both education and research ${ }^{4}$. See Figure 1. The EPSL includes six experiment stations with configurable loads, motor drives, meters, relays and controllers. The lab also includes a local area network (LAN), programmable logic controller (PLC) equipment, surge and sag generators, power factor correction capacitors, uninterruptible power supply (UPS), hardware development tools, smart meters and other test equipment. Both 480V AC and 208V AC systems are available, along with a DC system. The six test benches provide a total $30 \mathrm{~kW}$ of adjustable RLC load, including harmonic-producing compact fluorescent lights. A $25-\mathrm{kW}$ synchronous generator 
has been acquired for dedicated micro-gridding in the EPSL. The lab also contains six power quality monitors ${ }^{19}$ for undergraduate students to use in this course.

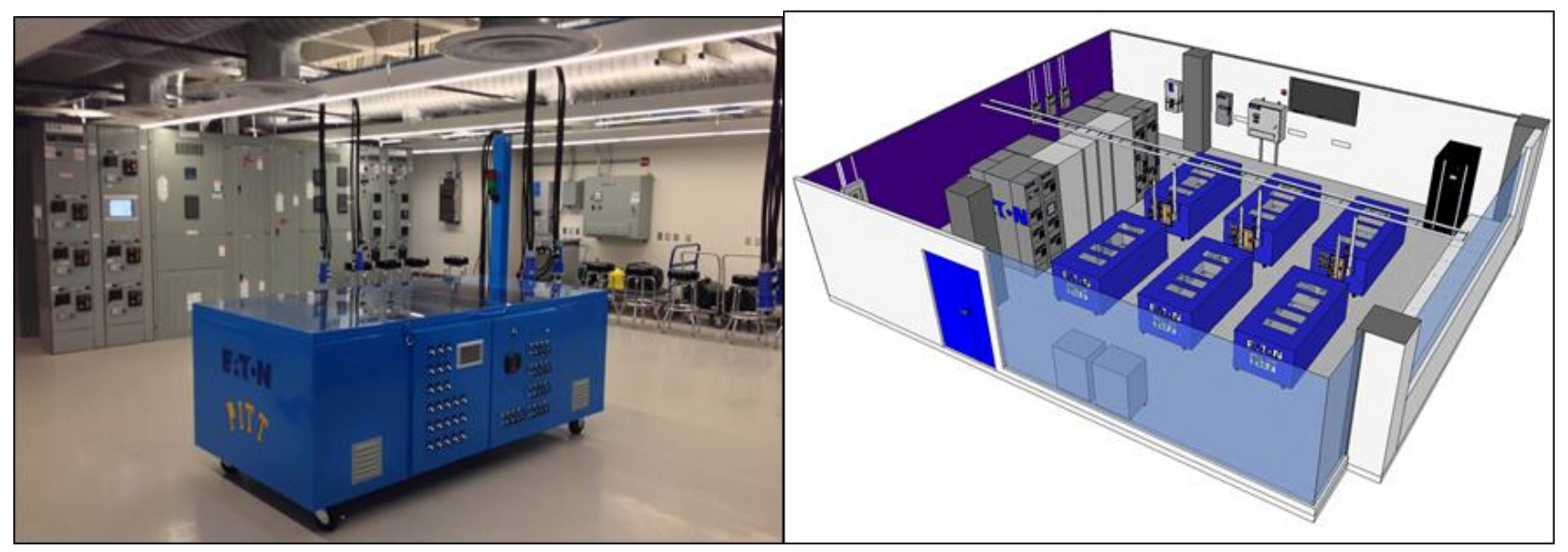

Figure 1: Electric Power Systems Laboratory, with one of six test benches delivered in October 2013 (left) and fully built out in January 2014 (right).

In 2014, the hardware lab assignments included:

1. Lab safety, basic monitor operation through measurement of compact fluorescent lighting harmonics, motor inrush current and motor no-load test (also done in 2013)

2. Power quality measurements: timed recordings and statistical analysis of harmonics, load switching identification, computer-based processing of results

3. Induction motor control with variable frequency drive: effects of source impedance and power factor correction capacitors, across-the-line (ATL) vs. reduced voltage starting system (RVSS), slip tests

4. Power quality measurements: capacitor switching, voltage sags, transient frequency analysis

5. Synchronous machine characteristics: DC resistance, open-circuit and short-circuit tests, loading tests at unity power factor

The first four experiments took advantage of the configurable lab bench (one-line diagram in Figure 2) to perform load switching, capacitor switching, motor starting, and motor operational tests. The bench load switches and monitoring points helped to reduce the lab setup time. The power quality monitors captured all three phase voltages and currents simultaneously, with several display modes:

1. Tabular display of metered quantities

2. Oscilloscope mode to display waveforms

3. Phasor display

4. RMS magnitude vs. time

5. FFT-based histograms of harmonic magnitudes 
The students learned how to set up the power quality monitor triggering scheme for each experiment. This was especially tested in experiment \#4, as the instructor generated voltage sags and swells, with different magnitudes on each phase, at unannounced times. Each team was able to capture and identify the various power quality events. The six lab groups finished their setup, data collection and tear-down at different times, but usually all groups were finished within 90 minutes. The one exception occurred when a group accidentally disconnected a motor drive keypad, and they had to wait for another group to finish before completing their data collection. Even then, all groups were out of the lab by 9:30 p.m. Data was transferred from the power quality monitors to PCs at the end of each lab, so that each group could finish their reports.

Experiment \#5 was performed at a regional campus approximately 2 hours driving time from our main campus. The equipment is shown in Figure 3-left. We couldn't do this experiment on the main campus, because we have not retained any synchronous machines for student use in the new lab. This experiment was optional, but over half the class chose to take a Saturday bus field trip from 9 a.m. until 4:30 to do this lab. The setup, data collection, and tear-down took a full four and a half hours using manual methods such as the two wattmeter method. Data was recorded manually and the students still had to complete their reports back on the main campus. However, based on the survey results and comments made at the time, this experience is valuable and will be kept in future course offerings.

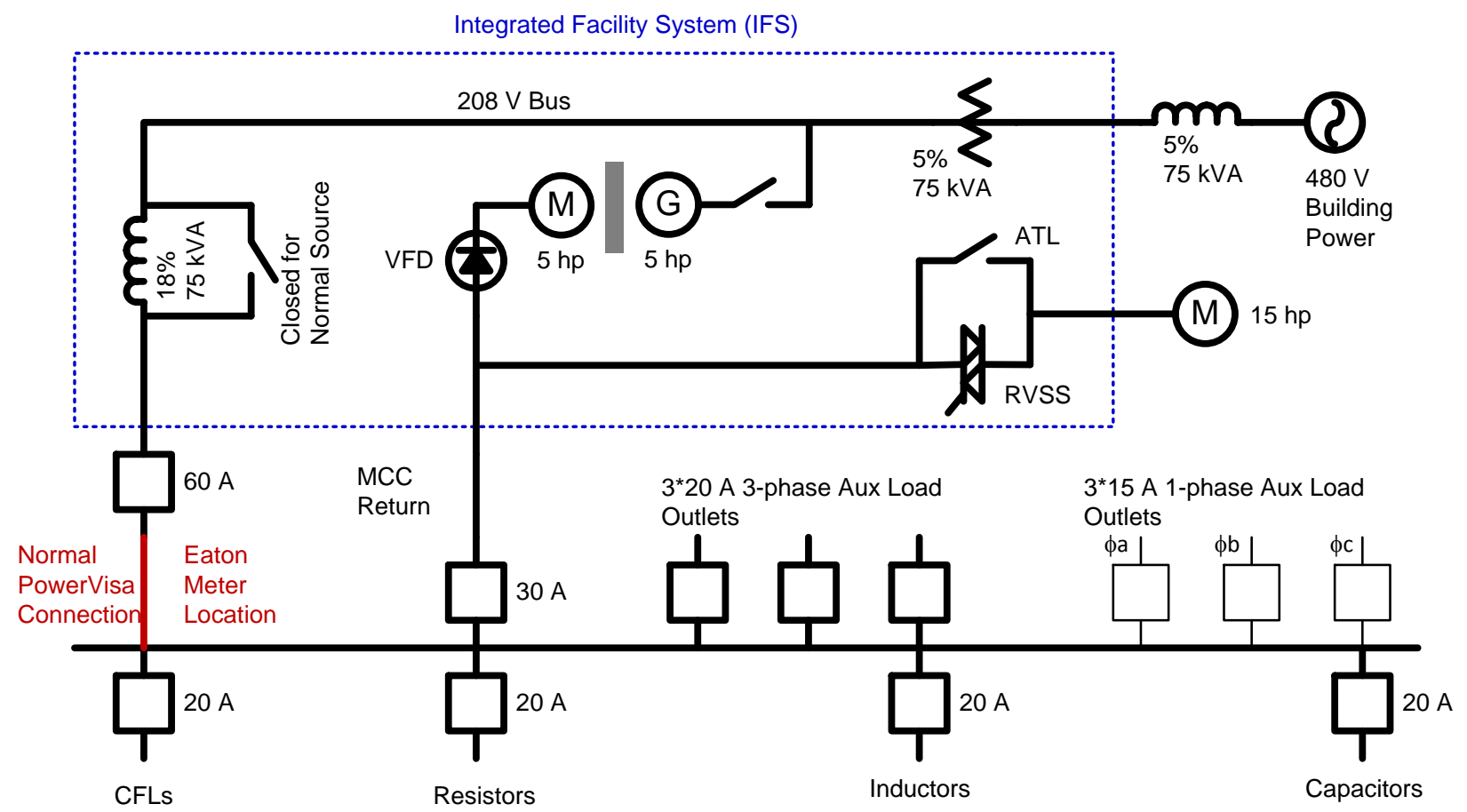

Figure 2: Partial One-line Diagram of a Lab Bench Interfaced to Motor Drives

Another optional field trip was provided to the local electric utility's high-voltage test facility. Students were able to witness testing of personal protective equipment and current transformers. 
They also inspected the inside of a tap changer, and witnessed a simulated medium-voltage distribution conductor contacting vegetation (Figure 3-right). The students thought this was "awesome" and worth repeating in future course offerings.
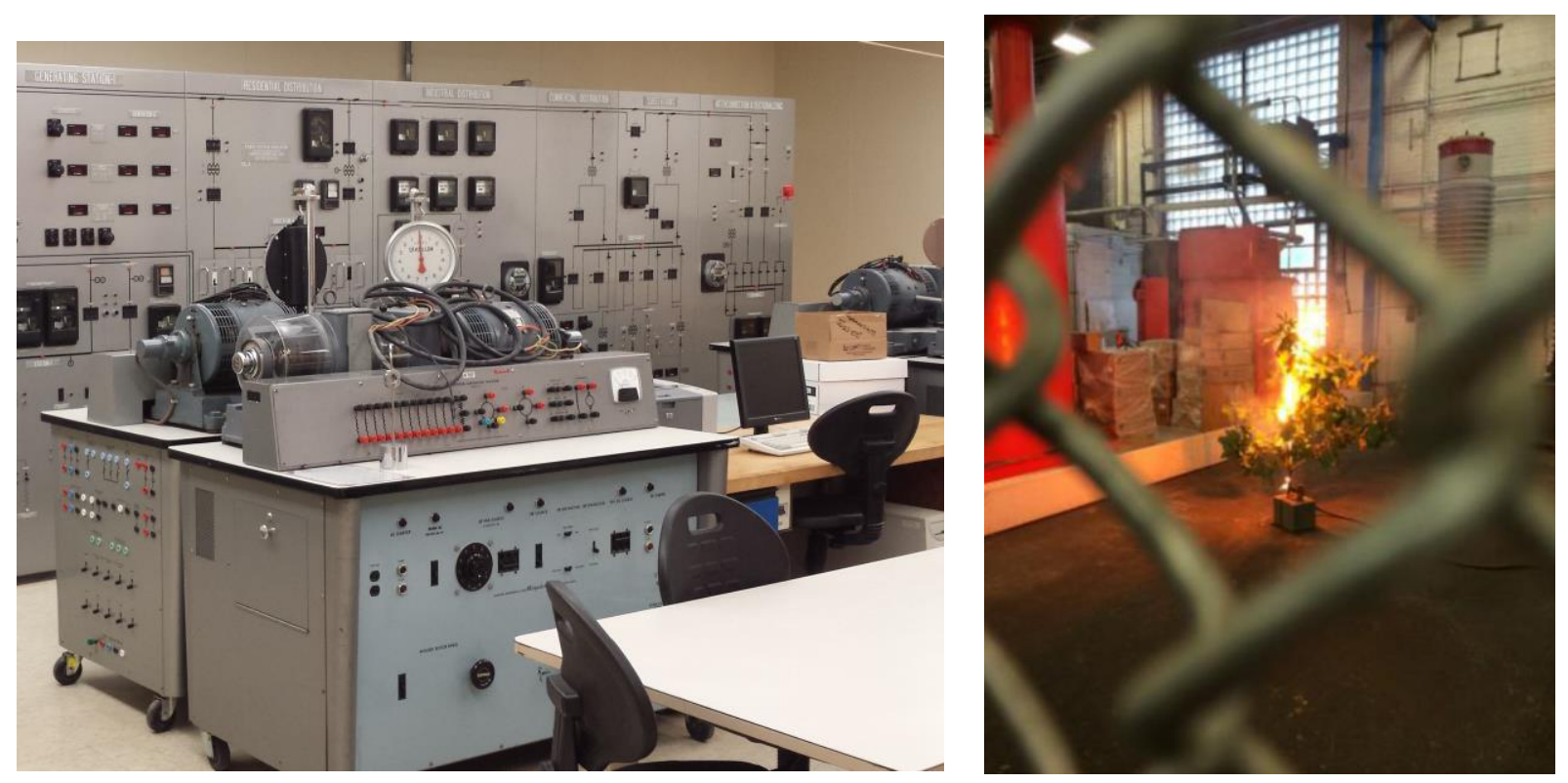

Figure 3: Field trips to the Universal Machine Benches at a Regional Campus (left) and the local utility's 250-kV test facility (right)

We originally planned to conduct a sixth hardware lab experiment on three-phase transformer connections, but the new transformer test banks were not ready in time. Instead, this experiment was done in software during the fall 2014 term.

\section{Software Lab Assignments}

The software lab assignments in fall 2014 included:

1. Finite element modeling of a 2D linear actuator with cylindrical symmetry in Maxwell

2. Parametric analysis of material and geometry properties of the force and inductance in a 2D linear actuator

3. Power electronics and control system modeling in Simplorer

4. System performance analysis of a $2 \mathrm{D}$ linear actuator with textbook and finite elementbased models, see Figure 4

5. Brushless DC machine performance analysis from geometry and material properties; generation of a finite element model from RMxprt, see Figure 5

6. Power electronics drive and control of a brushless DC machine

7. Three-phase transformer connections in Simplorer: connection of single-phase transformers in wye-wye, wye-delta, delta-wye and auto configurations to achieve proper phase shifts. Open-circuit and short-circuit tests. 
The first six experiments were also done in fall 2013. Experiment \#7, on three-phase transformer connections, was only done in fall 2014 and it will be performed in the new hardware lab starting in fall 2015. Based on a summer 2014 senior design project, and using single-phase transformers that were salvaged from the old lab, we are constructing new transformer banks on carts with wiring panels to support this experiment \#7, and also to support research in the lab. This was the most complicated and lengthy software experiment, and will work better in the hardware lab.

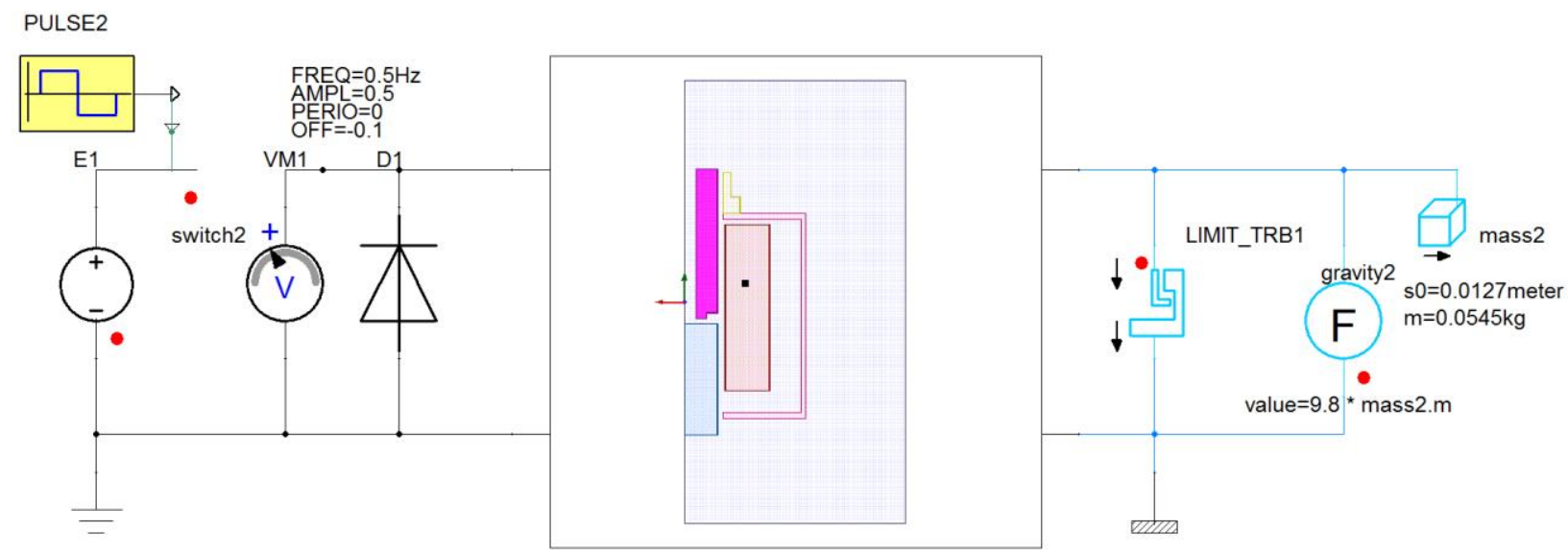

Figure 4: Finite Element Based Actuator Model Interfaced to a Drive Circuit and Load
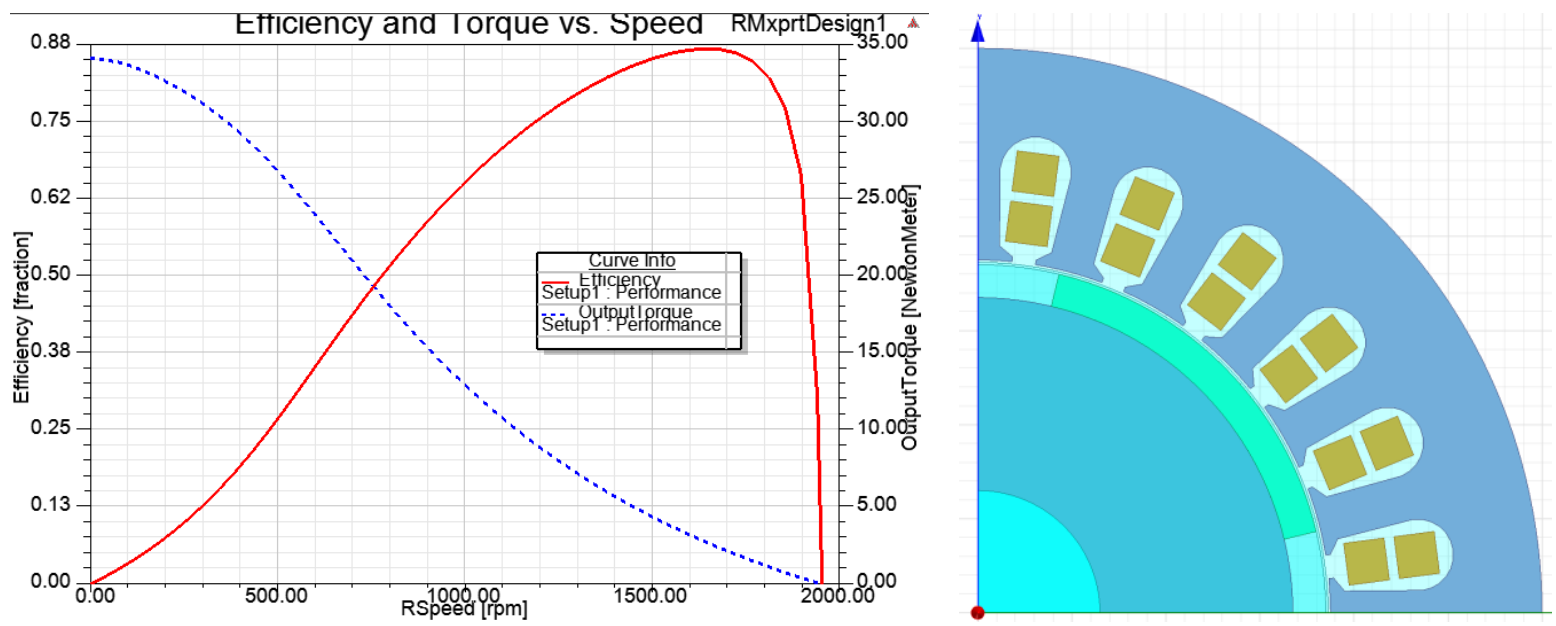

Figure 5: Brushless DC performance analysis (left) and finite element model (right)

\section{Classroom Observation in Fall 2014}

As a means to evaluate the flipped classroom via the activities of both the instructor and students, structured classroom observation was conducted in the fall 2014 semester using the Teaching Dimensions Observation Protocol (TDOP) ${ }^{20}$. This protocol involves a series of small observation windows (e.g., five-minute windows) and codes for recording teaching and learning behaviors. Classroom observation was done by the assessment analyst for the flipped classroom initiative in the school, who had previously established inter-rater reliability with a second 
observer using the TDOP. These observers were able to achieve an inter-rater reliability score of Cohen's $\kappa=0.86$ for the protocol as a whole. This kappa was based on observation of multiple flipped courses in the school; values of Cohen's kappa above 0.75 suggest strong agreement beyond chance. Classroom observation occurred at weeks four and nine of the term. The nonlab portion of the class period was observed in five-minute segments. In each segment, various activities and practices contained within our protocol were recorded as observed.

As shown in Table 4, the classroom was characterized by active learning by the students. In $45 \%$ of the observation segments, students were asked to work in small groups (SGW) to discuss (ART) and solve a problem (PS). Students actively asked questions (SCQ) in $32 \%$ of the observation segments. Many of the questions were asked during the problem solving session, as the instructor circulated among the students (MOV) to monitor progress and address students' questions. The instructor frequently assessed student understanding and progress in $26 \%$ of the segments observed. He also posed factual/conceptual questions in $28 \%$ of the segments, often seeking student responses by requesting a "vote" on a multiple-choice question. Student responses were noted in $30 \%$ of the observation segments. In addition to the active learning that occurred, the instructor lectured using the white board in $57 \%$ of the segments; thus, in the nonlab portion of this course, active problem solving occurred in about half of the in-class time.

Table 4: Comparison to 2012 National STEM TDOP Study (Two-Minute Window)

\begin{tabular}{clcc}
\hline & & \multicolumn{2}{c}{$\%$ of Observation Segments } \\
\hline $\begin{array}{c}\text { Classroom } \\
\text { Element }\end{array}$ & \multicolumn{1}{c}{ Description } & $\begin{array}{c}\text { STEM Comparison } \\
\text { Study }\end{array}$ & $\begin{array}{c}\text { Electric } \\
\text { Machinery }\end{array}$ \\
\hline SGW & Small group work & $\mathbf{9 \%}$ & $45 \% *$ \\
PS & Problem solving & $\mathbf{1 2 \%}$ & $45 \% *$ \\
ART & Student articulation/discussion & $\mathbf{9 \%}$ & $45 \% *$ \\
SCQ & Students ask question or request & $\mathbf{9 \%}$ & $32 \% *$ \\
MOV & assistance & & \\
\hline
\end{tabular}

$* p<0.0001$

$£ p<=0.05$

We compared our results to that of a recent TDOP observational study of 58 math and science faculty in three public research universities in order to understand the relative degree of active learning that occurred in our classroom ${ }^{21}$. This benchmark study used two-minute observation windows. The courses taught by these 58 instructors consisted of $38 \%$ upper and $62 \%$ lower division classes. We compared the percentages for each classroom element in Table 4 using Fisher's Exact test; Fisher's test can be used in lieu of a z-test of proportions when the numerators are small. The active learning elements of SGW, PS, ART, and SCQ were each significantly higher $(p<0.0001)$ in our electric machinery classroom versus in the 58 benchmark STEM classrooms. Although instructor circulation to monitor and assist was significantly higher 
in our classroom $(p=0.02)$, it would not be significant at $\alpha=0.05$ upon correcting for multiple comparisons using Bonferroni’s adjustment.

In a conversation with one of the TDOP developers, he pointed out that it's possible a fiveminute observation window could result in higher proportions relative to a two-minute window in certain cases ${ }^{22}$. For example, assume problem solving (PS) was recorded just once in ten minutes using the two-minute observation window. Its frequency of occurrence would be $20 \%$. However, using the five-minute window, the frequency would be $50 \%$. In this particular case, problem solving (PS) occurred rather infrequently during the ten minutes. In comparison, if PS had occurred continuously during that time, the frequencies of occurrence would have been the same at $100 \%$. In our flipped classroom, our students often worked for a substantial period (i.e., longer than two minutes at a time) on problem solving in groups. Nonetheless, we decided to obtain earlier benchmark data from the TDOP developer, in which a five-minute observation window had been used. This data was collected in 2010 and involved 57 math and science instructors at three large research universities ${ }^{20}$. We used this data as a second benchmark, as shown in Table 5. However, the TDOP was at an early stage of development when the fiveminute window was used by this other research team and was a much different protocol compared to the present protocol. Since several of the codes in Table 4 did not exist in the earlier protocol, we could only benchmark certain elements, such as SGW and PS. Thus, while benchmarking our five-minute-window results against the 2012 two-minute-window study was not optimal as discussed, a benchmark comparison using the earlier five-minute-window study also presented difficulties, given the differences with the current instrument.

Table 5: Comparison to 2010 TDOP Study (Five-Minute Window)

\begin{tabular}{clcc}
\hline & & \multicolumn{2}{c}{$\%$ of Observation Segments } \\
\hline $\begin{array}{c}\text { Classroom } \\
\text { Element }\end{array}$ & \multicolumn{1}{c}{ Description } & STEM Comparison & $\begin{array}{c}\text { Electric } \\
\text { Machinery }\end{array}$ \\
\hline SGW & Small group work & Study & $45 \% *$ \\
PS & Problem solving & $\mathbf{5 \%}$ & $45 \%$ \\
\hline$*_{p}<0.0001$ & $\mathbf{3 1 \%}$ &
\end{tabular}

In comparing Table 4 and Table 5, the most notable difference was related to the problem solving (PS) element. Using the five-minute window as described in Table 5, the benchmark comparison of PS was not significantly different, although it was with the two-minute-window comparison in Table 4. However, using either the two-minute or five-minute benchmark study, our flipped classroom exhibited a significantly greater frequency of small group work compared to the STEM classrooms in these recent large-scale studies. 


\section{Direct Assessment of Learning}

To directly assess learning in the non-flipped versus flipped versions of the course, we compared the average final exam and lab scores of the two groups using an analysis of covariance, with the pre-course GPA (i.e., grade point average) serving as the covariate or control variable. The final exam was very similar between the two semesters, and the labs were software-based labs that were comparable between the two semesters. The instructor, who was also the grader for the exam and lab assignments, remained the same between the two semesters. As a comparison of the two groups, the average pre-course cumulative GPAs were not significantly different, with the students in the non-flipped group having a slightly higher GPA than those in the flipped group, based on a Mann-Whitney test $(p=0.48)$.

Interestingly, there was generally a decrease in scores from the non-flipped to flipped versions of the course. However, the sample sizes were not large. For the final exam, there was a decrease from $87 \%$ to $84.5 \%$ in the raw scores, and these were based on 13 and 21 students, respectively. Since the sample size in each group was not large, we ran both a parametric and a nonparametric analysis of covariance. The non-parametric version is known as Quade's Test ${ }^{23,24}$. Based on both tests, the difference in final exam scores was not significant, with Quade's Test resulting in $p=0.336$. The adjusted means for the final exam and the three software labs are shown in Table 6. These means were "adjusted" by the SPSS software using the average value of the covariate, as is typically done.

Table 6: Direct Assessment - Non-Flip vs. Flip

\begin{tabular}{lcccccc}
\hline & \multicolumn{2}{c}{$\begin{array}{c}\text { Difference } \\
\text { Non-Flip vs. Flip }\end{array}$} & \multicolumn{2}{c}{$\begin{array}{c}\text { Adjusted } \\
\text { Mean Score }\end{array}$} & \multicolumn{2}{c}{$\begin{array}{c}\text { Sample } \\
\text { Size }\end{array}$} \\
& $\begin{array}{c}\text { Parametric } \\
\text { ANCOVA } \\
\boldsymbol{p}\end{array}$ & $\begin{array}{c}\text { Non-Parametric } \\
\text { ANCOVA } \\
\boldsymbol{p}\end{array}$ & Non-F & Flip & Non-F & Flip \\
\hline Final Exam & 0.262 & 0.336 & 86.6 & 84.8 & 13 & 21 \\
\hline Lab \#1 & 0.711 & 0.897 & 8.8 & 9.1 & 13 & 21 \\
\hline Lab \#2 & $<0.0005$ & $<0.0005$ & 9.4 & 8.8 & 12 & 21 \\
\hline Lab \#3 & 0.995 & 0.085 & 8.995 & 8.990 & 13 & 19 \\
\hline
\end{tabular}

Labs 2 and 3 also showed a decrease in score from the non-flipped to the flipped versions of the course, with lab \#2 showing a significant decrease, based on both tests $(p<0.0005)$. Only lab \#1 showed an increase, but this increase was not significant $(p=0.897)$. In the flipped classroom, the students prepared for the labs via the videos. In an end-of-course semi-structured interview, the instructor also noted that he had not noticed increases in problem solving ability, deep learning, or quality of the students' work with the flipped classroom. In a required junior-level electromagnetics course at the University of Utah, exam scores likewise showed no significant differences using the flipped format ${ }^{9}$. However, in a signal processing course at the University 
of Wisconsin, there was a very clear improvement in student achievement with the flipped classroom, with an overwhelming majority of students performing at a high level (i.e., above $70 \%$ ) on the final exam, as never seen before ${ }^{6}$. Thus, the available direct assessment results in flipped electrical engineering classrooms are mixed.

\section{Indirect Assessment of Learning}

The students' self-assessment of their learning, as determined via the end-of-course teaching evaluation, coincided with these direct assessment findings. In the non-flipped course, students rated their learning at 4.4 on the 1 to 5 scale $(n=9)$, with 5 representing "more learning" relative to 1 . However, in the flipped version of the course, the students rated their learning at 3.2 $(n=13)$. We ran a comparison of these means and found a significant difference between them based on both a $t$-test $(p=0.006)$ and the non-parametric Mann-Whitney test $(p=0.02)$. However, as a caution, the sample sizes were small, and the assumptions of each test were not fully met.

As part of the end-of-course teaching evaluation in 2014, we asked the students their perceptions regarding flipped instruction in this course. Thirteen of the twenty-one students (62\%) provided feedback. Of the 13 students, three (23\%) preferred the flipped classroom, eight (62\%) did not prefer it, and two (15\%) were undecided. In an open-ended question about suggestions to improve the course in general, four of the ten respondents suggested not using the flipped style of instruction, which coincides somewhat with the previous result. However, the students nonetheless saw value in flipped instruction, as $77 \%$ of the 13 respondents (i.e., 10 students) agreed or strongly agreed that they preferred problem solving or active learning in class with the instructor present versus listening to a lecture. The students reported having taken responsibility for the self-directed portion of the flipped classroom in large part, watching an average of $76 \%$ of the available videos. In addition, $69 \%$ of the students (i.e., 9 students) watched the videos before the class period for which they were assigned. The instructor did notice that some students were watching the videos on their mobile devices during class; thus, these data points suggest that not all students will take responsibility for watching the videos, even in a seniorlevel elective course. The in-class, team-based problem sets did serve to drive some accountability for this, and the instructor was in general pleased with the performance on these in-class active learning assignments. In an open-ended question about the benefits of flipped instruction, the students identified the ability to re-watch videos ( 2 students), problem solving during class ( 2 students), flexibility (1 student), and reinforcement of concepts (1 student). In a second open-ended question about drawbacks and suggestions relative to the flipped classroom, two students expressed dislike of the flipped classroom, and three provided suggestions about the use of in-class time, including more worked example problems during class ( 2 students).

\section{Instructor Reflections}

Although the results do not indicate significant improvements in achievement on exams and lab assignments with the flipped classroom, the instructor nonetheless found some positives in this 
method of instruction for the course. It enabled students to spend more time in the hardware lab, and five of the ten general comments about beneficial aspects of the course on the evaluation survey mentioned laboratory work. The hardware lab has modern equipment, which the students likely felt would help them in their future careers. Also, the lab environment was very exciting and dynamic, with much activity occurring, as the instructor and TA circulated among the teams to assist them with various obstacles. The instructor believes the flipped method of instruction requires students to talk to and explain things to one another and in general gets them engaged during class. These are important outcomes that we as educators often do not formally assess. Perhaps if we were to perform more "authentic" assessment at the student level in the flipped classroom and formally measure these types of learning objectives, we might identify significant differences in various behavioral learning outcomes with the flipped classroom.

\section{Conclusions}

The student course evaluations decreased significantly from 2013 to 2014, and for that reason alone, the instructor would not repeat this delivery method in the same way. Comparing the two sections, one of the most obvious differences in 2014 was the reduction in class lecture time to explain new concepts. The lab work also increased significantly in 2014, but the students viewed that positively. The flipped classroom enabled this increased time in the lab. Despite the lack of significant improvement in the exam or lab scores with the flipped classroom, the instructor nonetheless noted other positive outcomes, such as increased student interaction and engagement during class, which was also observed as part of the structured classroom observation using the TDOP.

Some of the new elements from 2014 should be useful in fall 2015 and future offerings. The video segments appear to be a good way of presenting additional sample problems, and the instructor is currently testing this more limited use of video in a spring 2015 section of our Linear Circuits and Systems 2 course. The instructor is also testing the use of short pre-lecture quizzes in the learning management system; these were not used in the fall 2014 course subject evaluation. Students in 2014 found the two optional field trips to be enjoyable and useful, so those will be kept.

We are adding a new undergraduate course in power electronics for the 2015-2016 school year. This will fill an important gap in our Electric Power Concentration, and it means that the electromechanical energy conversion course need not cover so much power electronics material. In turn, that allows the computer lab assignments to re-focus on electromechanical devices, as projected in Table 7. This will provide both hands-on hardware labs, and machine design content in two new software labs. Two of the fall 2014 software labs will be dropped, and another one moved to the hardware lab.

Most importantly, we plan to schedule the 2015 offering according to the more traditional schedule. There will be two 1:15 lecture periods per week, plus a 2-hour hardware or computer 
lab session each week. Distributing the activity should help foster student engagement and learning, with less of the interleaving topic presentations illustrated in Table 3.

Table 7: Planned Lab Assignments for Fall 2015

\begin{tabular}{|c|l|l|}
\hline ID \# & Hardware Lab & Software Lab \\
\hline 1 & Safety and measurements & Linear actuator modeling \\
\hline 2 & Transformer connections (new in hardware) & Parametric analysis of linear actuator \\
\hline 3 & Power quality: harmonics & System performance of linear actuator \\
\hline 4 & Power quality: switching and voltage sags & Transformer leakage flux paths (new) \\
\hline 5 & Induction motor control with VFD & Induction motor design (new) \\
\hline 6 & Synchronous generators (regional campus) & Brushless DC motor design \\
\hline
\end{tabular}

\section{Bibliography}

1. Grainger, B. M. and Reed, G. F., Methods and Approaches for Developing the Future Leaders of the Electric Power and Energy Industries, ASEE Annual Conference and Exposition, Indianapolis, IN, June 15-18, 2014.

2. Chapman, S., Electric Machinery Fundamentals, McGraw-Hill, 2011.

3. Hampden Universal Laboratory Machine (2015, Feb. 2) [Online]. Available: http://www.hampden.com/product-details.php?viewid=1020

4. Grainger, B. M., Barchowsky, A., Lewis, P. T., Cardoza, A. D., Reed, G. F, \& Carnovale, D. J., Electric Power Laboratory Workbench for Training the Next Generation of Engineering Professionals, 2014 IEEE T\&D PES Conference and Exposition, Chicago, IL, April 14-17, 2014.

5. Gross, C. A., Electric Machines, CRC Press, 2006.

6. Van Veen, B., Flipping Signal-Processing Instruction. IEEE Signal Processing Magazine, 30(6), 2013, pp. 145150 .

7. Bland, L., Applying Flip/Inverted Classroom Model in Electrical Engineering to Establish Life-Long Learning. Proceedings of the ASEE Annual Conference and Exposition, Chicago, IL, 2006.

8. Bachnak, R., \& Maldonado, S., A Flipped Classroom Experience: Approach and Lessons Learned. Proceedings of the ASEE Annual Conference and Exposition, Indianapolis, IN., 2014.

9. Furse, C., Lecture-Free Engineering Education. IEEE Antennas and Propagation Magazine, 53(5), 2011, pp. 176-179.

10. Wagner, D., Laforge, P., \& Cripps, D., Lecture Material Retention: a First Trial Report on Flipped Classroom Strategies in Electronic Systems Engineering at the University of Regina. Proceedings of the Canadian Engineering Education Association, Montreal, Quebec, 2013.

11. Riaz, M., Animation of Electric Machines (2015, Feb. 2) [Online]. Available: http://www.ece.umn.edu/users/riaz/animations/listanimations.html 
12. Mathcad M15 (2015, Feb. 2) [Online]. Available: http://www.ptc.com/product/mathcad

13. University of Pittsburgh Classes in Electric Power (2015, Feb. 2) [Online]. Available: https://www.youtube.com/channel/UCgTEq1cfVXOEO7sKgtZbdUw

14. Snagit (2014, Feb. 2), [Online] Available: http://www.techsmith.com/snagit.html

15. Smoothdraw 4 (2015, Feb. 2) [Online]. Available: http://www.smoothdraw.com/product/

16. Intuos Pen Small Tablet (2015, Feb. 2) [Online]. Available: http://www.wacom.com/en-es/products/pentablets/intuos-pen

17. Adobe Captivate 8 (2015, Feb. 2) [Online]. Available: http://www.adobe.com/products/captivate.html

18. ANSYS Electromechanical (2015, Feb. 2) [Online]. Available: http://www.ansys.com/Products/Simulation+Technology/Electronics/Electromechanical

19. Dranetz PowerVisa (2015, Feb. 2) [Online]. Available: http://www.dranetz.com/product-services/powervisa/

20. Hora, M., \& Ferrare, J., Instructional Systems of Practice: a Multidimensional Analysis of Math and Science Undergraduate Course Planning and Classroom Teaching. Journal of the Learning Sciences, 22(2), 2013, pp. 212-257.

21. Hora, M., Ferrare, J., \& Oleson, A., Findings from Classroom Observations of 58 Math and Science Faculty. Research Report: Culture, Cognition, and Evaluation of STEM Higher Education Reform NSF \# DRL-0814724, 2012.

22. Personal Communication with Matthew T. Hora, Ph.D., Researcher, Wisconsin Center for Educational Research, University of Wisconsin-Madison, Madison, Wisconsin, December 2014.

23. Quade, D., Rank analysis of covariance. Journal of the American Statistical Association, 62(320), 1967, pp. 1187-1200.

24. Lawson, A., Rank analysis of covariance: alternative approaches. The Statistician, 32(3), 1983, pp. 331-337. 